\title{
Perda de Resolução em Inversão Gravimétrica imposta numericamente por mal condicionamento, e uma solução.
}

\author{
William Pareschi Soares, IFPA, Brasil, Martins, C. M., UFPA, Silva, J. B. C., UFPA, Barbosa, V. C. F., ON.
}

Copyright 2014, SBGf - Sociedade Brasileira de Geofísica

Este texto foi preparado para a apresentação no VI Simpósio Brasileiro de Geofísica, Porto Alegre, 14 a 16 de outubro de 2014. Seu conteúdo foi revisado pelo Comitê Técnico do VI SimBGf, mas não necessariamente representa a opinião da SBGf ou de seus associados. É proibida a reprodução total ou parcial deste material para propósitos comerciais sem prévia autorização da SBG.

\section{Resumo}

Apresentamos um novo procedimento de restituição da resolução na inversão gravimétrica linear para o mapeamento da distribuição de densidade de pequenas fontes, que possibilita novas perspectivas em aplicações de gravimetria, inclusive para estudos ambientais. Parte da resolução que é fisicamente possível de ser recuperada pode ser perdida devido a fenômeno numérico computacional que leva a estimativas tendenciosas e muito suaves. Esta condição é numericamente imposta se as dimensões horizontais das fontes são pequenas. Contornamos este problema numérico com uma abordagem semiheurística que estende as dimensões horizontais do modelo intrepretativo, e posteriormente, corrige as estimativas. Apresentamos a metodologia para estimar, via inversão gravimétrica regularizada, a distribuição de contraste de densidade de fontes gravimétricas de pequenas dimensões. Com este novo procedimento obtivemos em testes sintéticos o delineamento de contatos laterais entre regiões de diferentes densidades, que só seriam possíveis de se recuperar com fontes de dimensões maiores.

\section{Introdução}

A inversão gravimétrica linear pode ser aplicada com o objetivo de mapear a distribuição de densidade em subsuperfície, mapear o relevo do embasamento de bacias sedimentares ou delinear a forma de corpos intrusivos, mas em todos os casos, as estimativas do conjunto de parâmetros do modelo escolhido são obtidas a partir dos dados gravimétricos e informações adicionais conhecidas a priori. Esta obtenção do modelo geofísico interpretado e a recuperação de detalhes das fontes geológicas estão relacionadas à resolução do problema gravimétrico inverso.

Contudo, diferentes autores tratam de diferentes aspectos da resolução. BACKUS e GILBERT (1968; 1970), e PARKER (1977) mostraram o importante compromisso entre a resolução e estabilidade da solução. Mesmo fazendo uso do método de regularização (TIKHONOV e ARSENIN, 1977) para a obtenção de soluções únicas e estáveis em problemas gravimétricos inversos, a falta de resolução pode ser inevitável.

Segundo SILVA et al. (2010) é preciso distinguir entre "resolução verdadeira" e "resolução demandada". A primeira é a menor distância entre duas fontes geológica, ou pontos na distribuição da propriedade física, na qual estas fontes podem ser distinguidas pelos dados geofísicos, enquanto que resolução demandada é a resolução que o geofísico espera obter dos dados. Note que a informação a priori introduzida pelo funcional regularizador (regularização de TIKHONOV) bem como o peso atribuído numericamente a regularização (valor atribuído ao parâmetro de regularização de TIKHONOV) não são capazes de simultaneamente aumentar a resolução verdadeira e manter a estabilidade. Presumindo-se uma determinada função regularizadora e um determinado peso para esta função podemos obter uma solução única e estável. Porém, um peso pequeno atribuído à função regularizadora aumenta a resolução demandada, mas também aumenta substancialmente a instabilidade da solução estimada. Por outro lado, um peso grande atribuído à função regularizadora estabiliza a solução, mas reduz a resolução demandada. A instabilidade aparece quando a resolução demandada torna-se maior que a resolução verdadeira o que pode ser interpretado como uma tentativa de se extrair mais informações dos dados do que é fisicamente possível. Portanto a sintonia de um valor para o parâmetro de regularização pode levar na melhor das hipóteses apenas à máxima resolução fisicamente possível.

Ocorre que parte dessa resolução fisicamente possível de ser recuperada pode ser perdida devido a fenômeno numérico computacional que leve ao mal condicionamento da matriz sensibilidade ao quadrado. Matematicamente a matriz Resolução é calculada a partir da matriz de sensibilidade ao quadrado. Como abordado por BOTT (1972), se os valores singulares da matriz sensibilidade ao quadrado se aproximam de zero ocorre um empobrecimento de seu condicionamento (ANDERSEN, 1969), que afeta sua inversa e leva a estimativas tendenciosas e muito suaves. Verificamos que este mal condicionamento da matriz sensibilidade ao quadrado, e a consequente perda de resolução, são impostos numericamente se as dimensões horizontais das fontes são pequenas.

Contornamos este problema numérico com uma abordagem semi-heurística que consiste de duas etapas. Primeiro, estendemos as dimensões horizontais do modelo interpretativo utilizado na inversão gravimétrica para estimar provisoriamente os parâmetros, assim não há mal condicionamento da matriz sensibilidade ao quadrado. No entanto, como o volume das fontes foi exagerado, o valor da propriedade física é subestimado. Em seguida, corrigimos a magnitude destes valores subestimados utilizando o desajuste com a anomalia gravimétrica observada.

Apresentamos a metodologia para estimar via inversão gravimétrica regularizada a distribuição de contraste de densidade de fontes gravimétricas de pequenas dimensões. 


\section{Metodologia}

Seja horizontalmente heterogênea a distribuição de densidade de um pacote de aterro depositado sobre um embasamento de densidade homogênea. Discretizamos este aterro em uma malha $m x \times m y$ de $M$ prismas elementares 3D, verticais e justapostos nas direções horizontais $x$ e $y$ em um sistema destral de coordenadas em que $z$ é positivo para baixo. O topo dos prismas coincide com a superfície do aterro e todos os prismas têm dimensões horizontais iguais à $d x$ e $d y$ ao longo das direções $x$ e $y$, respectivamente.

Presumimos que o contraste de densidade $\left(\Delta \rho_{j}\right)$, entre um prisma e o embasamento, é constante dentro de cada um dos $M$ primas, mas pode mudar de um prisma para o outro a fim de permitir variação na distribuição horizontal de densidade do aterro. As espessuras $\left(t_{j}\right)$ dos $M$ prismas são conhecidas e representam as profundidades do embasamento do aterro em $M$ pontos.

A componente vertical do sinal gravimétrico no $i$-ésimo ponto de observação $\left(g_{i}\right)$, nas coordenadas $x_{i}, y_{i}$ e $z_{i}$, está relacionada ao contraste de densidade do $j$-ésimo prisma $\left(\Delta \rho_{j}\right)$ através da relação linear

$g_{i}=\sum_{j=1}^{M} f_{i}\left(\Delta \rho_{j}, t_{j}, d x, d y, x_{j}, y_{j}\right), j=1, \ldots, M$,

sendo a função linear $f_{i}\left(\Delta \rho_{j}, t_{j}, d x, d y, x_{j}, y_{j}\right)$ calculada no $i$-ésimo ponto de observação para o $j$-ésimo prisma com centro localizado nas coordenadas horizontais $x_{j}$ e $y_{j}$.

A solução algorítmica da integral que define a função $f_{i}$ é dada por PLOUFF (1976):

$$
\begin{aligned}
f_{i}=\gamma \Delta \rho_{j} \sum_{k=1}^{2} \sum_{l=1}^{2} \sum_{m=1}^{2} \mu_{k l m}\left[z_{m} \arctan \frac{x_{k} y_{l}}{z_{m} R_{k l m}}\right. \\
\left.-\log \left[\left(R_{k l m}+y_{l}\right)^{x_{k}}\left(R_{k l m}+x_{l}\right)^{y_{k}}\right]\right]
\end{aligned}
$$

sendo,

$\mu_{k l m}=(-1)^{k}(-1)^{l}(-1)^{m}$,

$$
R_{k l m}=\sqrt{x_{k}^{2}+y_{l}^{2}+z_{m}^{2}},
$$$$
x_{1}=\left(x_{i}-x_{j}\right)-d x / 2 \text {, }
$$

$x_{2}=\left(x_{i}-x_{j}\right)+d x / 2, y_{1}=\left(y_{i}-y_{j}\right)-d y / 2, y_{2}=\left(y_{i}-\right.$ $\left.y_{j}\right)+d y / 2, z_{1}=0$ e $z_{2}=t_{j}$, para $z_{i}=0$.

O termo $g_{i}$ (equação 1) define o $i$-ésimo elemento do vetor $\boldsymbol{g}^{\boldsymbol{o}} \equiv\left(g_{1}^{o}, \ldots, g_{M}^{o}\right)^{T}$, contendo a anomalia teórica computada através do modelo interpretativo definido de modo único pelo vetor de parâmetros $\boldsymbol{p} \equiv\left(\Delta \rho_{1}, \ldots, \Delta \rho_{M}\right)^{T}$, e o sobrescrito $T$ representa transposição.

Por simplicidade, presumimos que os dados gravimétricos observados são interpolados em uma malha regular $m x \times m y$ com espaçamentos $d x$ e $d y$ ao longo das direções $x$ e $y$, respectivamente, e que a posição horizontal do centro de cada prisma coincide com um ponto de observação (Figura 1).

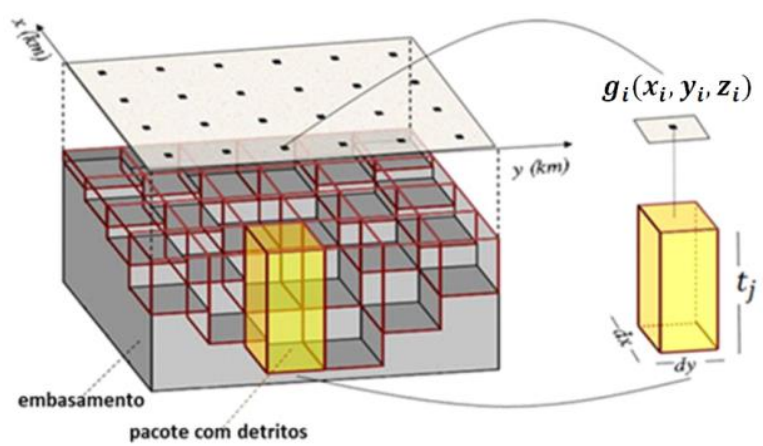

Figura 1: Modelo interpretativo sem extensão - Parte inferior - Pacote de aterro discretizado por uma malha $m x \times m y$ de $M$ prismas $3 \mathrm{D}$, de dimensões horizontais $d x \mathrm{e}$ $d y$, justapostos ao longo das direções $x$ e $y$, respectivamente; Parte superior - Malha regularmente espaçada com as posições das observações; Detalhe a direta - Contribuição gravimétrica do $j$-ésimo prisma na observação $g_{i}$ posicionada sobre o centro do $j$-ésimo prisma.

A inversão gravimétrica consiste em estimar, a partir de $\boldsymbol{g}^{\boldsymbol{o}}$, o vetor de parâmetros $\widehat{\boldsymbol{p}}$ contendo a distribuição do contraste de densidade $\Delta \rho_{j}$ dos $M$ prismas que compõe o modelo interpretativo. Estes contrastes de densidade estão relacionados aos dados gravimétricos através da relação linear dada pela equação 1 . O problema inverso linear de estimar $\hat{\boldsymbol{p}}$ a partir dos dados gravimétricos pode ser formulado, apenas utilizando-se mínimos quadrados, segundo MEDEIROS e SILVA (1996), como:

$\widehat{\boldsymbol{p}}=\left(\boldsymbol{A}^{T} \boldsymbol{A}\right)^{-1} \boldsymbol{A}^{T} \boldsymbol{g}^{\boldsymbol{o}}$.

em que, $\boldsymbol{A}$ é a matriz sensibilidade, cujo $i j$-ésimo elemento é definido como:

$$
\begin{aligned}
\boldsymbol{A} \equiv\left\{a_{i j}\right\}=\frac{\partial f_{i}}{\partial p_{j}} & =\gamma \sum_{k=1}^{2} \sum_{l=1}^{2} \sum_{m=1}^{2} \mu_{k l m}\left[z_{m} \arctan \frac{x_{k} y_{l}}{z_{m} R_{k l m}}\right. \\
& \left.-\log \left[\left(R_{k l m}+y_{l}\right)^{x_{k}}\left(R_{k l m}+x_{l}\right)^{y_{k}}\right]\right]
\end{aligned}
$$

Note que se os termos $x_{k}$ e $y_{k}$ tenderem a zero, o resultado do logaritmo natural tende a zero, e que isso ocorre se $d x$ e $d y$ tenderem a zero.

O problema inverso linear de estimar um vetor $\widehat{\boldsymbol{p}}$ apenas utilizando a equação 2 é mal posto apresentando solução sem estabilidade. Assim, para transformar esse problema mal posto em outro bem posto usualmente um funcional regularizador é introduzido. Como presumimos que a distribuição horizontal do contraste de densidade é predominantemente suave, ao menos em uma mesma região, incorporamos esta informação a priori através da regularização conhecida como suavidade (regularizador Tikhonov de primeira ordem, TIKHONOV e ARSENI, 1977). Esta técnica impõe que a estimativa de cada parâmetro $\Delta \rho_{j}$ (contraste de densidade do $j$ ésimo prisma) se aproxime da estimativa dos parâmetros adjacentes. Segundo MEDEIROS e SILVA (1996) o estimador regularizado com o funcional de suavidade é dado por:

$\widehat{\boldsymbol{p}}=\left(\boldsymbol{A}^{T} \boldsymbol{A}+\mu \boldsymbol{B}^{T} \boldsymbol{B}\right)^{-1} \boldsymbol{A}^{T} \boldsymbol{g}^{\boldsymbol{o}}$,

que $\mu$ é um escalar não negativo denominado de parâmetro de regularização, $\boldsymbol{B}$ é uma matriz $L \times M$ 
representando o operador discreto de primeiras derivadas horizontais (TWOMEY, 1963), e $L$ é o número total de pares de parâmetros adjacentes. A $i$ ésima linha da matriz $\boldsymbol{B}$ contém somente dois elementos não nulos, 1 e -1 , que estão associados ao $i$-ésimo par de parâmetros adjacentes.

A matriz pseudo- inversa $\boldsymbol{H}$, é definida por

$\boldsymbol{H}=\left(\boldsymbol{A}^{T} \boldsymbol{A}\right)^{-1} \boldsymbol{A}^{T}$, ou por $\boldsymbol{H}=\left(\boldsymbol{A}^{T} \boldsymbol{A}+\mu \boldsymbol{B}^{T} \boldsymbol{B}\right)^{-1} \boldsymbol{A}^{T}$,

se é utilizada, respectivamente, a inversão descrita pela equação 3 ou pela equação 5 . Em ambos os casos, a Matriz Resolução $(\boldsymbol{R})$, segundo BACKUS E GILBERT (1970), fica definida por

$\boldsymbol{R}=\boldsymbol{H A}$.

Note que a realização da inversão descrita pela equação 3 implica na igualdade da matriz Resolução com a matriz Identidade $(\boldsymbol{R}=\boldsymbol{I})$. No entanto, quando as fontes prismáticas têm dimensões horizontais ( $d x$ e $d y$ ) pequenas isso não ocorre devido a uma degeneração numérica da matriz $\boldsymbol{A}^{T} \boldsymbol{A}$. Decompondo em valores singulares as matrizes $\boldsymbol{A}$ e $\boldsymbol{A}^{T} \boldsymbol{A}$ temos que $\boldsymbol{A}=\boldsymbol{U} \boldsymbol{S} \boldsymbol{V}^{T}$ e $\boldsymbol{A}^{T} \boldsymbol{A}=\boldsymbol{U} \boldsymbol{S}^{2} \boldsymbol{V}^{T}$. Fica claro que se os valores singulares forem próximos de zero, seu quadrado será ainda mais próximo de zero. Quando as dimensões horizontais das fontes prismáticas $(d x$ e $d y)$ são suficientemente grandes o termo relacionado com a função logarítmica natural da equação 4 leva a valores singulares grandes, por garantir uma soma aos elementos da diagonal da matriz Sensibilidade $(\boldsymbol{A})$. Por outo lado se $d x$ e $d y$ tendem a zero a matriz $\boldsymbol{A}^{T} \boldsymbol{A}$ ser torna mal condicionada (ANDERSEN 1969) e terá valores singulares próximos a zero. Adicionalmente, embora todos os elementos da matriz $\boldsymbol{A}^{T} \boldsymbol{A}$ sejam numericamente próximos de zero, relativamente os elementos da diagonal são muito maiores que os elementos fora da diagonal. Isto implica em uma simultânea suavidade e tendenciosidade ao vetor de parâmetros estimados $(\widehat{\boldsymbol{p}})$.

A fim de superar esta dificuldade numérica imposta às inversões que envolvam $\boldsymbol{A}^{T} \boldsymbol{A}$, propomos um procedimento de inversão que temporariamente modifica as dimensões do modelo interpretativo, estima os contrastes de densidade provisórios, e os corrige em uma etapa final. Multiplicamos por um fator $c$ as dimensões horizontais dos prismas, $d x$ e $d y$, e os espaçamentos horizontais entre as observações gravimétricas $\left(g_{i}^{o}\right)$ produzindo assim um novo modelo interpretativo horizontalmente estendido. Aplicamos a inversão gravimétrica regularizada (equação 5), no vetor de observações gravimétricas $\left(\boldsymbol{g}^{\boldsymbol{o}}\right)$ mas com espaçamento estendido entre as observações, para estimar um vetor de parâmetros provisório $\widetilde{\boldsymbol{p}}$ contendo as $M$ estimativas provisórias de contraste de densidade $\left(\Delta \tilde{\rho}_{j}\right)$ deste novo modelo interpretativo estendido. Com este procedimento os parâmetros $\Delta \tilde{\rho}_{j}$ são subestimados pela inversão como consequência do superdimensionamento do volume do modelo interpretativo estendido. No entanto, este procedimento é efetivo para localizar contatos, mapeando no pacote de aterro regiões lateralmente dispostas com diferentes contrastes de densidade. É possível recuperar a magnitude verdadeira dos valores da distribuição do contraste de densidade estimados multiplicando cada elemento do vetor de contraste de densidade provisório $\left(\tilde{p}_{i}\right)$ por um escalar $\left(\alpha_{i}\right)$, que funciona como fator de correção.

Ao calcularmos a anomalia gravimétrica produzida com o modelo interpretativo original, mas com a distribuição de densidade subestimada $\widetilde{\boldsymbol{p}}$, obtemos uma anomalia gravimétrica $\left(\breve{\boldsymbol{g}}^{c}\right)$ a qual apresenta forma aproximada à da anomalia gravimétrica observada $\left(\boldsymbol{g}^{\boldsymbol{o}}\right)$, mas amplitude inferior. Para a correção de $\widetilde{\boldsymbol{p}}$ calculamos um fator de correção $\left(f c_{i}\right)$ para cada $i$-ésimo contraste de densidade subestimado $\left(\tilde{p}_{i}\right)$ pela razão entre a $i$-ésima anomalia gravimétrica verdadeira $\left(g_{i}^{0}\right)$ e a $i$-ésima anomalia gravimétrica ajustada $\left(\breve{g}_{i}^{c}\right)$ :

$\alpha_{i}=\frac{\left|\boldsymbol{g}_{i}^{\boldsymbol{o}}\right|}{\left|\breve{\boldsymbol{g}}_{i}^{\boldsymbol{c}}\right|}$.

Finalmente, para obtermos o $i$-ésimo elemento do vetor de parâmetros estimado definitivo $\hat{p}_{i}$, multiplicamos o $i$-ésimo elemento do vetor de parâmetros estimado provisório $\tilde{p}_{i}$ pelo $i$-ésimo fator de correção $\alpha_{i}$,

$\widehat{p}_{i}=\tilde{p}_{i} \cdot \alpha_{i}$

\section{Resultados}

Simulamos um aterro em que o pacoterro simulado cobre um embasamento homogêneo com arcabouço estrutural complexo caracterizado por três baixos. A Figura 2 mostra o mapa de contorno e a vista em perspectiva do relevo verdadeiro do embasamento do aterro simulado. Este aterro estende-se por $130 \mathrm{~m} \mathrm{e}$ $160 \mathrm{~m}$ ao longo das direções $x$ e $y$ (norte-sul e lesteoeste) respectivamente, sendo composto por $26 \times 32$ prismas com dimensões horizontais $d x$ e $d y$ de $5 \mathrm{~m}$, todos com topo em $z_{1}=0,0 \mathrm{~m}$. A espessura de cada prisma $\left(t_{j}\right)$ é obtida na coordenada horizontal $x_{j}, y_{j}$, do centro do $j$-ésimo prisma, pela profundidade verdadeira do embasamento (Figura 2) nas mesmas coordenadas.

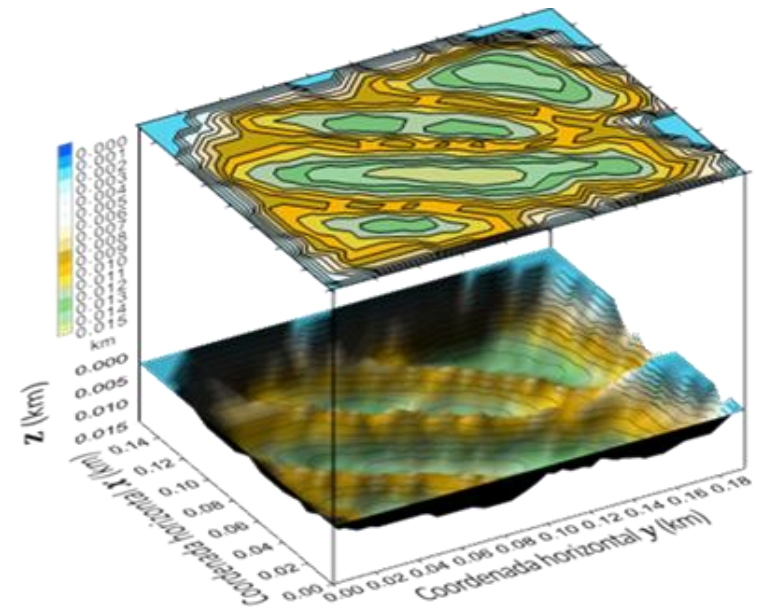

Figura 2: Mapa de contorno e vista em perspectiva do relevo verdadeiro do aterro simulado.

Simulamos uma distribuição horizontal do contraste de densidade, entre o embasamento e cada um dos prismas $\left(\Delta \rho_{j}\right)$ que compõem o aterro, dividida em três regiões distintas (Figura 3 ), regiões I, II e III, sendo a I a região com menor contraste de densidade. 


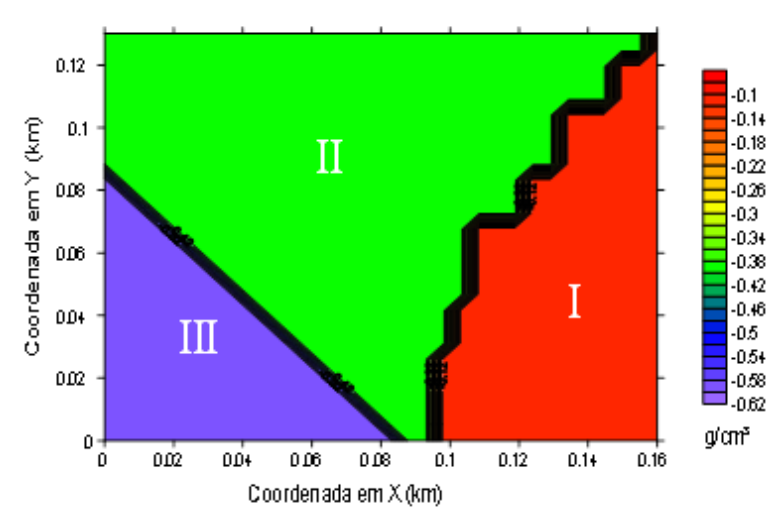

Figura 3: Mapa da distribuição do contraste de densidade para $o$ aterro simulado.

A Figura 4 mostra a anomalia gravimétrica, contaminada com ruído, produzida pelo modelo simulado, com relevo do embasamento mostrado na Figura 2 e a distribuição do contraste de densidade mostrado na Figura 3. A anomalia gravimétrica teórica (equação 1) foi avaliada nas mesmas coordenadas horizontais dos centros dos prismas, no plano horizontal $z_{o}=0,5 \mathrm{~m}$, e corrompida por ruído através de realizações de uma variável pseudo-aleatória Gaussiana com média nula e desvio padrão de 0,1 mGal.

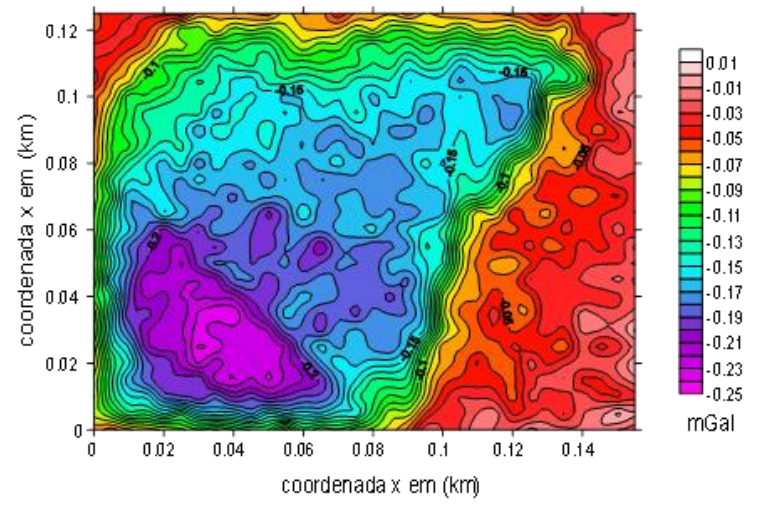

Figura 4: Anomalia gravimétrica do aterro simulado contaminada com ruído pseudoaleatório de $0.01 \mathrm{mGal}$.

\section{Inversão}

Inversão sem procedimento de extensão A Figura 5 mostra o mapa da distribuição do contraste de densidade estimado via inversão linear (equação 5) com parâmetro de regularização $(\mu)$ de $10^{-7}$ e modelo interpretativo sem extensão.

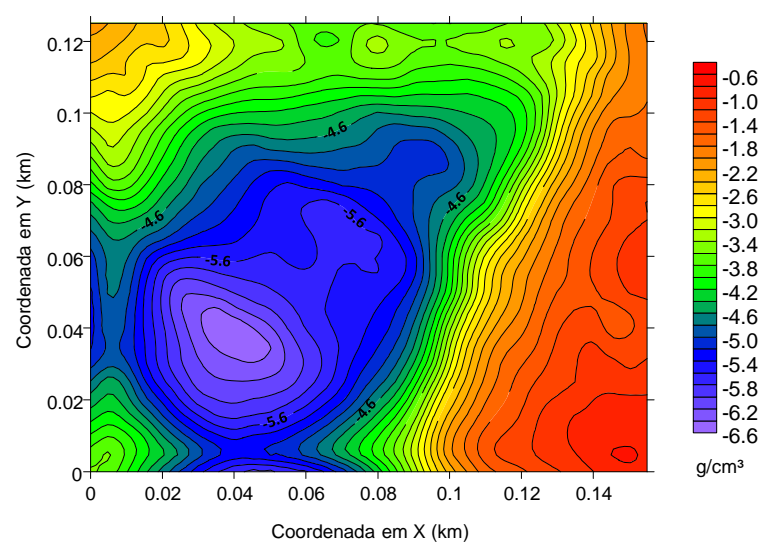

Figura 5: Mapa da estimativa do contraste de densidade via inversão linear sem extensão com parâmetro de regularização $(\mu)$ igual a $1 \cdot 10^{-7}$.

Note que a distribuição do contraste de densidade difere da verdadeira em dois aspectos, quanto a forma e quanto a amplitude. Note também que há uma intensa suavidade imposta à estimativa

A Figura 6 mostra a anomalia gravimétrica ajustada pela inversão linear sem extensão.

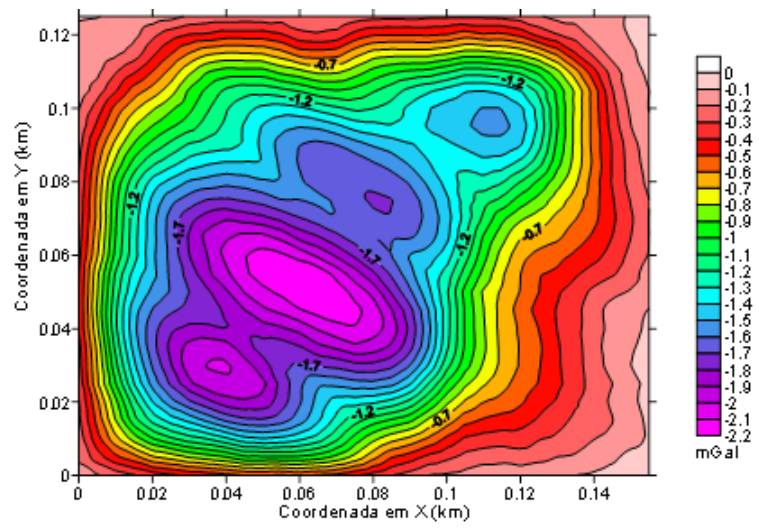

Figura 6: Anomalia gravimétrica ajustada pela inversão linear sem extensão.

Note que anomalia gravimétrica recuperada com inversão linear sem extensão (Figura 6) não se ajusta à verdadeira nem quanto a forma nem quanto a intensidade (Figura 4).

Inversão com procedimento de extensão

A Figura 7 mostra o mapa da distribuição do contraste de densidade estimado via inversão linear (equação 5) com parâmetro de regularização $(\mu)$ de $10^{-7}$ utilizandose o procedimento de extensão, com fator de extensão $c=100$. 


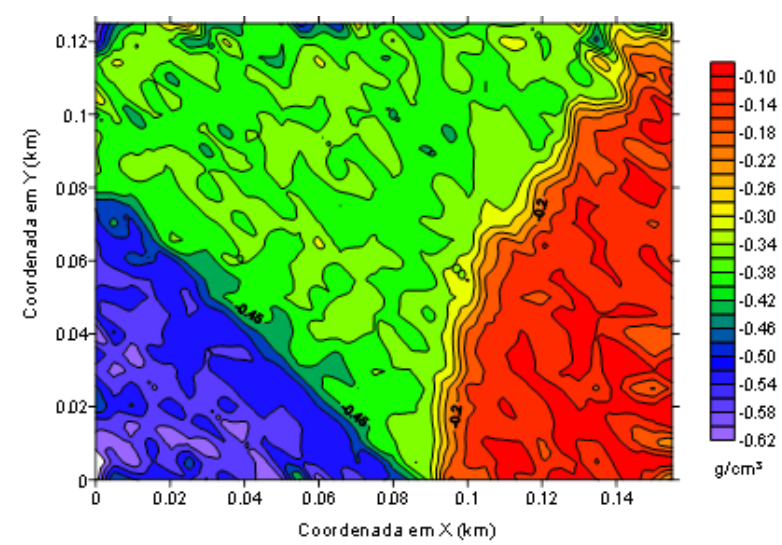

Figura 7: Mapa da estimativa do contraste de densidade via inversão linear com extensão com parâmetro de regularização $(\mu)$ igual à $1 \cdot 10^{-7}$ e fator de extensão $c=100$.

Note que utilizando à inversão linear com extensão a distribuição do contraste de densidade verdadeiro é muito bem recuperada.

A Figura 8 mostra a anomalia gravimétrica ajustada pela inversão linear com extensão.

Note que anomalia gravimétrica recuperada com inversão linear com extensão (Figura 8) se ajusta muito bem à verdadeira (Figura 4).

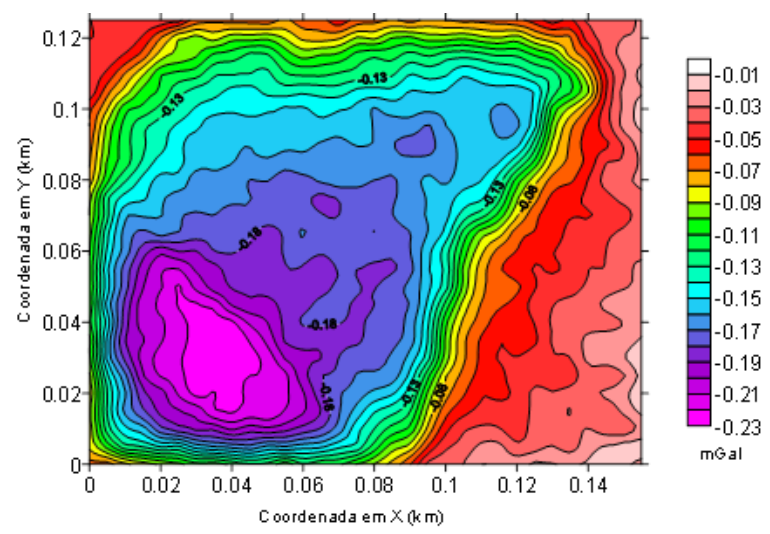

Figura 8: Anomalia gravimétrica ajustada pela inversão linear com extensão.

A Figura 9 mostra o resíduo entre a anomalia gravimétrica verdadeira (Figura 4) e a anomalia gravimétrica ajustada com a inversão linear com extensão.

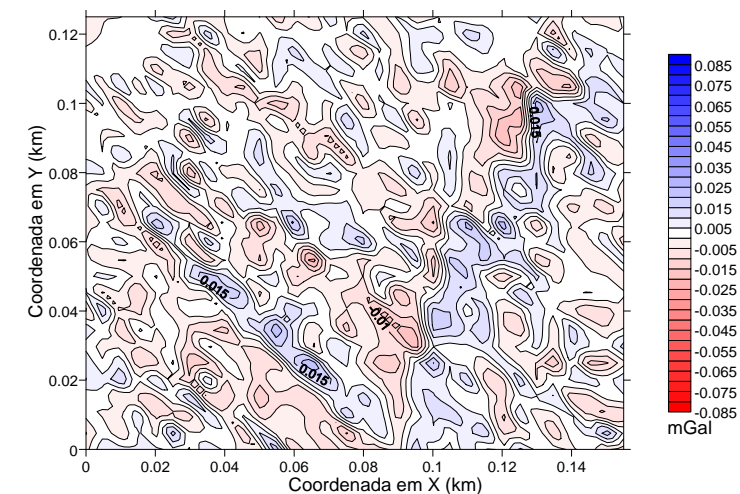

Figura 9: Mapa do resíduo entre a anomalia gravimétrica verdadeira e ajustada com a inversão linear com extensão.
A Figura 10 mostra o histograma da distribuição dos resíduos entre as anomalias verdadeira e ajustada com a inversão linear com extensão.

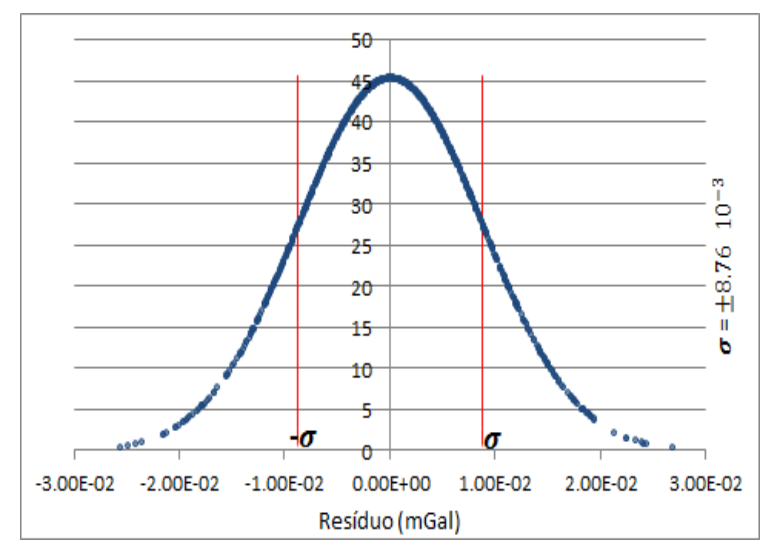

Figura 10: Histograma do resíduo entre a anomalia gravimétrica verdadeira e ajustada com a inversão linear com extensão, com desvio padrão $\sigma= \pm 8.7610^{-3}$.

O histograma da Figura 10 mostra que a distribuição do resíduo apresenta forma que se aproxima de uma distribuição Gaussiana, que corresponde à distribuição do ruído introduzido.

A Figura 11 mostra, para a inversão linear sem e com extensão, as matrizes de Resolução, apresentadas em mapas com curvas de contorno com valores interpolados para indicar em escala de corres os elementos das matrizes. Foram utilizando em ambos os casos o mesmo valor para parâmetro de regularização do vínculo de suavidade $\mu=10^{-7}$ e um fator de extensão $c=100$ para a inversão linear com extensão. Note que as escalas de cores em ambos os casos não são iguais, sendo a da inversão linear com extensão (Figura 11-b) a que apresenta os maiores valores.

(a)

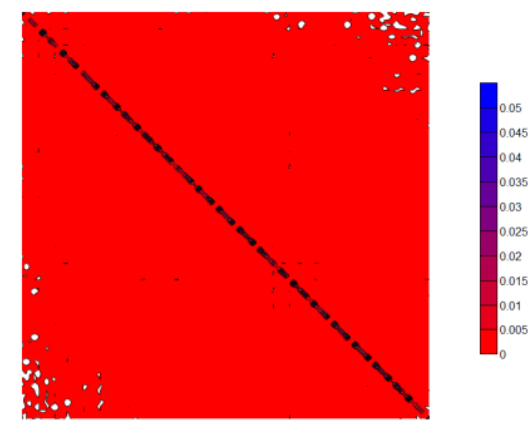

(b)

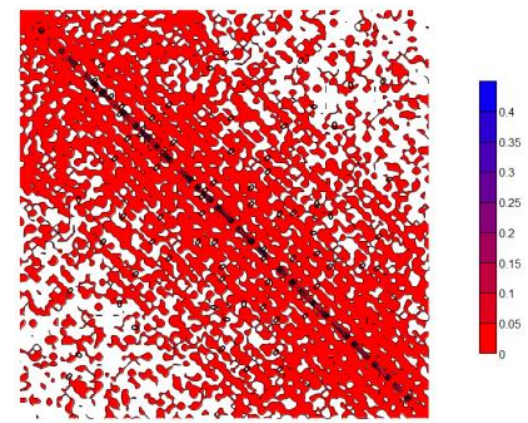

Figura 11: Matriz Resolução (equação 7) (a) da inversão linear sem extensão e (b) da inversão linear com extensão. 
Note que ambas as matrizes de Resolução na Figura 11 apresentam os maiores valores na diagonal principal, e valores fora da diagonal que se aproximam de zero. Mas, a matriz resolução da inversão linear com extensão (Figura 11-b) possui o valor de máximo de em sua diagonal aproximadamente 8 vezes maior que a matriz resolução da inversão linear sem extensão (Figura 11-a).

\section{Conclusão}

Caracterizamos que há degeneração numérica da matriz sensibilidade ao quadrado que leva a perda da Resolução em inversões gravimétricas que utilizem esta matriz para estimativas da propriedade física de fontes de pequenas dimensões horizontais, e apresentamos os resultados não efetivos dessa inversão.

Apresentamos um procedimento de inversão que envolve estender horizontalmente 0 modelo interpretativo e que contorna o problema numérico envolvendo a matriz sensibilidade ao quadrado.

Esta metodologia pode ser aplicada ao mapeamento da distribuição de contraste de densidade em corpos de pequenas dimensões, como em aterros sanitários por exemplo.

Outra possibilidade de aplicação deste novo método é - mapeamento geológico de detalhe a partir da estimativa da distribuição do contraste de densidade da camada superficial.

\section{Agradecimentos}

Os autores agradecem o apoio do Programa de PósGraduação em Geofísica da UFPA. C. M. M. agradece 0 apoio financeiro do CNPq/INCT-GP (processo 573890/2008-0). W. P. S. agradece o apoio do IFPA.

\section{Referências}

Anderssen, R. S., 1969, On the solution of certain overdetermined systems of linear equations that arise in geophysics, Jornal Geophys. Res., 74, 1045-1051.

Backus, G., and Gilbert, F., 1968. The resolving power of gross Earth data: Geophys. J. Royal Astron. Soc., 16, 169-205.

Backus, G., and Gilbert, F., 1970, Uniqueness in the inversion of inaccurate gross Earth data: Philos. Trans. Royal Soc. London, 266, 123-192.

Bott, M. H. P., Ingels, A., 1972, Matrix Methods for Joint Interpretation of Two-dimensional Gravity and Magnetic Anomalies with Aplication to the Iceland-Faeroe Ridge: Geophys. J. Royal Astron. Soc., 30, 55-67.

Medeiros, W. E. e Silva, J. B. C, 1996, Geophysical inversion using approximate equaly constraints: Geophysics, 61, $1678-1688$.

Parker, R. L., 1977, Understanding inverse theory: Annual Review of Earth and Planetary Sciences,5, 3564.
PLOUFF, D., 1976, Gravity and magnetic fields of polygonal prisms and application to magnetic terrain corrections: Geophysics, v.41, p.727-41.

Silva, J. B. C., Oliveira, A. S., Barbosa, V. C. F., 2010, Gravity inversion of 2D basement relief using entropic regularization: Geophysics, 75, 129-135.

Tikhonov, A. N., and Arsenin, V. Y., 1977, Solutions of ill-posed problems: W. H. Winston \& Sons.

Twomey, S.,1963, On the numerical solution of Fredholm integral equations of the first kind by the inversion of the linear system produced by quadrature: Journal of the Association for Computing Machinery, 10, 97-101. 